\title{
Tribbles pseudokinase 3 (TRIB3) contributes to the progression of hepatocellular carcinoma by activating the mitogen-activated protein kinase pathway
}

\author{
Rui-Qi Wang $^{1 \#}$, Fa-Zhong He ${ }^{1 \# \wedge}$, Qian Meng ${ }^{1}$, Wei-Jie Lin ${ }^{1}$, Jia-Mei Dong ${ }^{1}$, Hai-Kui Yang ${ }^{1}$, Yang Yang ${ }^{1}$, \\ Min Zhao ${ }^{1}$, Wen-Tao Qiu ${ }^{1}$, Yong-Jie Xin ${ }^{2}$, Zhi-Ling Zhou ${ }^{1}$ \\ ${ }^{1}$ Department of Pharmacy, Zhuhai People's Hospital, Zhuhai Hospital Affiliated with Jinan University, Zhuhai, China; ${ }^{2}$ Zhuhai Interventional \\ Medical Center, Zhuhai Precision Medical Center, Zhuhai People's Hospital, Zhuhai Hospital Affiliated with Jinan University, Zhuhai, China \\ Contributions: (I) Conception and design: RQ Wang, FZ He; (II) Administrative support: ZL Zhou, YJ Xin; (III) Provision of study materials or \\ patients: M Zhao, WJ Lin, JM Dong, WT Qiu; (IV) Collection and assembly of data: Q Meng, Y Yang; (V) Data analysis and interpretation: RQ \\ Wang, HK Yang, JM Dong; (VI) Manuscript writing: All authors; (VII) Final approval of manuscript: All authors. \\ \#These authors contributed equally to this work. \\ Correspondence to: Zhi-Ling Zhou. Department of Pharmacy, Zhuhai People's Zhuhai Hospital Affiliated with Jinan University, Zhuhai, China. \\ Email: zhouzhiling@ext.jnu.edu.cn; Yong-Jie Xin. Zhuhai Interventional Medical Center, Zhuhai Precision Medical Center, Zhuhai People’s \\ Hospital, Zhuhai Hospital Affiliated with Jinan University, Zhuhai 519000, China. Email: xinyongjie55@163.com.
}

Background: Tribble pseudokinase 3 (TRIB3) plays a key role in regulating the malignancy of many tumors. This study examined its function in cancer cells and explored the potential mechanisms of action.

Methods: The expression of TRIB3 was examined in hepatocellular carcinomas (HCCs) using The Cancer Genome Atlas (TCGA) database. A TRIB3 lentivirus with a flag label was constructed and transfected into Huh7 and Hep3B human hepatoma cell lines to generate cells that stably overexpress TRIB3. A small interfering RNA (siRNA) was designed to knockdown TRIB3 mRNA in HepG2 and Huh7. Cell viability and cell colony formation assays were conducted. Flow cytometry was performed to assess the cell cycle in cells overexpressing TRIB3. Western blotting were performed to examine the expression of (Mitogen-activated protein kinase, MAPKK) (MEK), phosphorylated-MEK (p-MEK), extracellular signal-regulated kinase (ERK), and p-MEK in cells with TRIB3 knockdown. The correlation between TRIB3 and SMARCD 3 was assessed using co-immunoprecipitation assays and immunofluorescence.

Results: TRIB3 was significantly overexpressed in advanced grade HCC tissues and was closely correlated with poor prognosis. TRIB3 overexpression promoted the cell growth and cell cycle but had little effect on migration capabilities in Huh7 and Hep3B cells. Conversely, knockdown of TRIB3 had slow down the cell growth in Huh7 and HepG2 cells detected by CCK8 and colony formation assay. The expression of $M E K$ and $E R K$ at both the protein and mRNA levels were downregulated when TRIB3 was knocked down. The protein expression of $p-E R K$ and $p-M E K$ were also downregulated upon TRIB3 silencing. SMARCD 3 is a transcript factor that is belongs to the SWI/SNF complex and has been shown to regulate many genes. Indeed, co-immunoprecipitation assays demonstrated that TRIB3 interacts with SMARCD3 in the nucleus, suggesting that it may regulate TRIB3 in HCCs.

Conclusions: This study demonstrated that TRIB3 promotes the malignancy of HCC cells and its expression may be a potential diagnostic biomarker for HCC progression.

Keywords: Tribble pseudokinase 3 (TRIB3); hepatocellular carcinoma (HCC); extracellular signal-regulated kinase (ERK)

^ ORCID: Rui-Qi Wang, 0000-0002-4075-8815; Fa-Zhong He, 0000-0001-5750-0135. 
Submitted Jun 10, 2021. Accepted for publication Aug 05, 2021.

doi: $10.21037 / \mathrm{atm}-21-2820$

View this article at: https://dx.doi.org/10.21037/atm-21-2820

\section{Introduction}

Hepatocellular carcinoma (HCC) is one of the most common primary liver cancers and a leading cause of cancer-related deaths globally $(1,2)$. The etiology of HCC is multifactorial and may include viral infections, dietary and environmental toxins, carcinogens such as aflatoxin and aristocratic acid, and chronic and excessive alcoholism. In addition, non-alcoholic fatty liver disease associated with HCC may result from obesity caused by a poor lifestyle such as lack of physical activity or exercise. The pathogenesis of HCC is complex and has been shown to be directly related to excessive intake of a high-calorie diet, insulin resistance, endoplasmic reticulum stress, oxidative stress, and obesity (3). The development and progression of HCC involves multiple signaling pathways such as proliferation, invasion, migration, and resistance to apoptosis stimulation. Tumor proliferation is one of the principal markers of tumorigenesis and it is related to the growth, development, and metastasis of HCC. The mitogen-activated protein kinase/extracellular signal-regulated kinase (MAPK/ERK) pathway, also known as the Ras-RAF-MEK-ERK pathway, is involved in the $\mathrm{HCC}$-associated tumor proliferation by transmitting mitogenic signals from the cell surface transmits to the nucleus $(4,5)$. However, to date, alphafetoprotein (AFP) is the only diagnostic tumor marker available for assessing the prognosis and drug resistance in patients with HCC. Hence, the identification or development of a more accurate and specific tumor marker for HCC is wanting (6).

Tribble pseudokinase 3 (TRIB3) is 358 amino acids long and has a molecular weight of $39.6 \mathrm{kD}$. Transcription and translation of the TRIB3 protein is mediated by the transcription factor NF-кB. TRIB3 has three different functional motifs, namely, the central kinase-like domain, and the nitrogen-terminal and carbon-terminal protein binding domains with different functional motifs $(7,8)$. TRIB 3 serves as a key "stress regulator" connecting homeostasis, metabolic disease, and cancer, and in fact, some studies have suggested that TRIB 3 may act as a biomarker of disease and as a therapeutic target (7). The "pseudokinase" function of TRIB3 results in the inactivation of multiple transcription factors and signaling proteins (9). Abnormal expression of TRIB 3 has been associated with poor prognosis in colorectal, prostate, and breast cancer, and high expression of TRIB3 in gastric cancer has been associated with vascular endothelial growth factor receptor (VEGFR) expression and tumor vascular density (10-14). High glucose levels can promote the proliferation and migration of non-small cell lung cancer (NSCLC) and decreased the degree of TRIB3 ubiquitination (15). The most well-studied role of TRIB3 has been in the PI3K/Akt/mTOR pathway. Upregulation of TRIB3 in endometrial cancer can inhibit tumor progression, and cancer cells can upregulate AKT by inhibiting the expression of TRIB3 (16). The upregulation of TRIB3 expression can also interfere with the interaction between microtubule-associated protein $1 \mathrm{~A} / 1 \mathrm{~B}$-light chain 3 (LC3) and the ubiquitin-binding protein p62/SQSTM1 to promote tumorigenesis. Furthermore, the MEK1 binding domain of TRIB3 enables it to inactivate multiple MAP kinases (17). However, the precise molecular mechanisms by which TRIB3 promotes HCC are still poorly understood and remain to be elucidated.

To explore the molecular mechanisms of TRIB 3 in HCC, the effects of TRIB 3 on cell proliferation, colony formation, cell cycle, and cell migration were examined in HCC cells. TRIB3 promoted HCC cell proliferation and cycle progression, and played a role in the regulation of HCC characteristics through the MAPK signaling pathway. Therefore, TRIB 3 may be a potential biomarker for the diagnosis of HCC or a potential therapeutic target for patients with HCC. We present the following article in accordance with the MDAR reporting checklist (available at https://dx.doi.org/10.21037/atm-21-2820).

\section{Methods}

\section{TCGA data}

High throughput RNA sequencing data from 369 samples and matched normal tissues in the TCGA database and 160 samples from the Genotype-Tissue Expression (GTEx) project were obtained. The differences in gene expression were analyzed by GEPIA 2. The cut-off is based on the median value of 182 pairs of mRNA expression levels in cancer and adjacent. The association between overall 
survival (OS) and TRIB3 expression was identified.

\section{Cell culture}

The human hepatoma cell lines, Hep3B, Huh7, HepG2, and HCCLM3 were obtained from ZqxzBio (Shanghai, China). LO2 and $97 \mathrm{H}$ were provided by the Precision Treatment Center of Zhuhai People's hospital. LO2 is normal liver cell lines, the others are cancer cells. All the Cells were cultured in high glucose Dulbecco's modified Eagle's medium (DMEM) supplemented with $10 \%$ fetal bovine serum, $100 \mu \mathrm{g} / \mathrm{mL}$ streptomycin, and $100 \mu \mathrm{g} / \mathrm{mL}$ penicillin at $37^{\circ} \mathrm{C}$ and $5 \% \mathrm{CO}_{2}$. Mycoplasma contamination was excluded in all cells. All procedures performed in this study involving human participants were in accordance with the Declaration of Helsinki (as revised in 2013). Written informed consent has been obtained from each patient and the study was approved by the ethics committee of Zhuhai People's Hospital, Zhuhai Hospital Affiliated with Jinan University.

\section{Cell transfection}

The lentiviral vector containing the human TRIB 3 sequence was synthesized by (Hanbio Biotechnology Co. Ltd., Shanghai, China). The TRIB3-specific small interfering RNA (siRNA) oligonucleotides were synthesized by IGEbio Co., Guangzhou, China). A total of $1 \times 10^{6}$ cells were seeded into 6-well plates and transfected with the specific siRNA using iMAX reagent (Thermo Fisher technologies, California, USA) according to the manufacturer's instructions. After 48 hours, cells were harvested. The siRNA and primer sequences used in this study are provided in Table S1.

\section{Cell proliferation assay}

A total of $1 \times 10^{3}$ cells were seeded into a 96-well plate and cell proliferation rate was assessed each day. Cells were cocultured with $10 \mu \mathrm{L}$ Cell Counting Kit 8 (CCK-8) solution for 2 hours at $37^{\circ} \mathrm{C}$. Absorbance was measured at $450 \mathrm{~nm}$ using a VARIOSKAN LUX microplate reader (Thermo Fisher technologies, California, USA).

\section{Western blot analysis}

Western blot was used to assess TRIB3 protein expression as previously described (18). Briefly, whole cell lysates were prepared and quantified using RIPA reagent. Protein samples were separated using sodium dodecylsulfate polyacrylamide gel electrophoresis (SDS-PAGE) and transferred onto nitrocellulose membranes (BioRad). Membranes were incubated in a blocking solution, followed by incubation with rabbit anti human monoclonal antibody against TRIB3 (1:1,000, ab137526, Abcam, USA), SMARCD3 (SWI/SNF related, matrix associated, actin dependent regulator of chromatin, subfamily $\mathrm{D}$, member 3; 1:1,000, ab171075,Abcam, USA), or glyceraldehyde 3-phosphate dehydrogenase (GAPDH; 1:5,000, Abcam, USA) for 18 hours at $4{ }^{\circ} \mathrm{C}$. Membranes were then washed three times with Tris-buffered saline Tween (TBST) and incubated with a goat anti-rabbit IgG-AP secondary antibody (1:5,000 dilution, \#HA1019, HuaBio, Hangzhou, China) in room temperature for 1 hour. After washing, the proteins on the membrane were detected by an enzyme labeling method and chemiluminescence in accordance with the manufacturer's instructions (Omni ECL reagent (EpiZyme, Shanghai, China).

\section{Flow cytometry assay}

For cell cycle the cells transfected by TRIB3 was harvested and analyzed by Cell Cycle and Apoptosis Analysis Kit (YEASEN biological Co., Ltd., Shanghai, China) following the manufacturer's protocols. Briefly, cells were harvested and fixed with $95 \%$ cold ethanol for 18 hours at $4{ }^{\circ} \mathrm{C}$. After removal of the ethanol by low-speed centrifugation, the cells were resuspended in phosphate buffered saline (PBS) and centrifuged again. Then the cells were incubated with propidium iodide (PI) in buffer solution for 30 minutes at $37^{\circ} \mathrm{C}$ and analyzed by an 8-color 3-laser FACSCanto II flow cytometer (BD Biosciences, USA).

\section{Immunofluorescence}

A total of $4 \times 10^{3}$ cells were seeded on special dishes for laser confocal scanning. Cells were fixed with $4 \%$ paraformaldehyde at room temperature for 30 minutes, prestabilized with $0.1 \%$ Triton X-100 in PBS for 15 minutes, and blocked with $5 \%$ bovine serum albumin at room temperature for 1 hour. Cells were then incubated with a primary antibody at $4{ }^{\circ} \mathrm{C}$ for 18 hours. Following three 5 -minute washes with PBST (0.1\% Tween-20 in PBS), cells were incubated with TRIB3 antibody (anti-TRIB3, \#ab75846, 1:400 dilution, Abcam, Cambrige, USA) a fluorescent conjugated secondary antibody $(1: 1,000$, CST). 
Cells were then treated with 4',6-diamidino-2-phenylindole (DAPI; YEASEN, Shanghai, China) and stored in the dark and visualized with a Confocal laser endomicroscopy (Carl Zeiss Meditec AG, Jena, Germany).

\section{Co-immunoprecipitation assay}

Co-immunoprecipitation (co-IP) assays were performed with magnetic beads according to the manufacturer's instructions (YEASEN, Shanghai, China). Briefly, cell lysates were incubated with protein $\mathrm{A} / \mathrm{G}$ magnetic beads pre-bound to the primary antibody for 18 hours. The magnetic beads were then removed to dissociate the bound antigen from the antibody cross-linked beads. The eluate was detected by protein analysis.

\section{Statistical analysis}

All statistical analyses were carried out using SPSS software (version 23.0, SPSS Corporation, Chicago, Illinois, USA) and Graphpad prism software (version 8.0, Jolla, California). All data are shown as mean \pm standard deviation. The Students' $t$-test was used to investigate continuous variables. The chi square test was used to test correlation. Survival curves were drawn using the by Kaplan-Meier method and tested by the log rank test. All analyzes were two-tailed and $\mathrm{P}<0.05$ was considered statistically significant. All in vitro experiments were performed in triplicates.

\section{Results}

The expression of TRIB3 was upregulated in HCC samples

Based on analysis of the GEPIA 2.0 database, TRIB 3 was found to be highly expressed in most tumors (Figure $1 \mathrm{~A}$, $\mathrm{P}<0.01)$. The expression of TRIB3 in HCC tumor tissues was significantly higher than that in normal liver tissues from the TCGA datasets (Figure $1 B, \mathrm{P}<0.01$ ). Oncomine studies also found that the expression of TRIB3 in liver hepatocellular carcinoma (LIHC) tissues was significantly higher than that in normal tissues, as previously reported in other investigations, including Guichard Liver and Roessler Liver (Figure 1C,1D, $\mathrm{P}<0.05)$. The GEPIA database was used to determine the prognostic value of TRIB3. As shown in Figure 1E, the overall survival (OS) of patients with high TRIB3 expression was inferior to that of patients with low TRIB3 expression of $[\mathrm{P}<0.05$; hazard ratio (HR) (high) $=1.5 ; \mathrm{N}=182]$. The data from the K-M plotter database also revealed that
TRIB3 was correlated with poor prognosis $[\mathrm{P}=0.0012$; HR (high) $=1.58 ; \mathrm{N}=364]$ (Figure 1F). These data suggested that TRIB3 may be a good prognostic marker of HCC.

TRIB3 knockout inhibited the growth of HCC cells, but had little impact on the migration ability

Immunohistochemistry was conducted on a HCC tissue array to examine TRIB3 expression at different stages of HCC and in the adjacent healthy tissues (Figure $2 A$ ). These results suggested that the expression of TRIB 3 increased with the advancement of HCC stage. The expression of TRIB3 in HCC was assessed by RT-qPCR and Western blot analyses. As shown in Figure 2B, the expression of TRIB3 was significantly higher in the HepG2 and Huh7 cell lines compared to the other cell lines. These cell lines originated from patients who had HCC, but were not infected with the hepatitis B virus (HBV), and thus they represent a good model for non-virus infected HCC. Immunofluorescence demonstrated that TRIB3 was mainly localized to the cytoplasm with little expression in the nucleus of HepG2 cells (Figure 2C).

\section{Overexpression of the TRIB3 gene promotes the proliferation and cell cycle progression of HCC cells}

To further elucidate the role of TRIB3 in the growth and metastasis of HCC, a lentiviral vector was used to stably overexpress TRIB 3 in the Huh7 and Hep3B cell lines. These cell lines show relatively low endogenous levels of TRIB3. Furthermore, a specific siRNA targeting TRIB3 (siTRIB3) was used to suppress TRIB3 expression in the Huh7 and HepG2 cells. The transfection efficiency was assessed by RT-qPCR and Western blot (Figure 2D,2E).

Overexpression of TRIB3 promoted cell proliferation and colony formation, while cells transfected with siTRIB 3 showed inhibited proliferation and colony formation (Figure $3 A, 3 B$ ). In addition, cell cycle experiments showed that overexpression of TRIB 3 decreased the G0/G1 proportion and increased the $\mathrm{S}$ and G2/M fractions in Hep3B cells (Figure 3C). Interestingly, overexpression of TRIB3 did not promote the migration of tumor cells (Figure S1). TRIB3 inhibits the progression of HCC through the MAPK
signaling patbway

Analysis of Huh7 cells overexpressing TRIB3 and control 
A

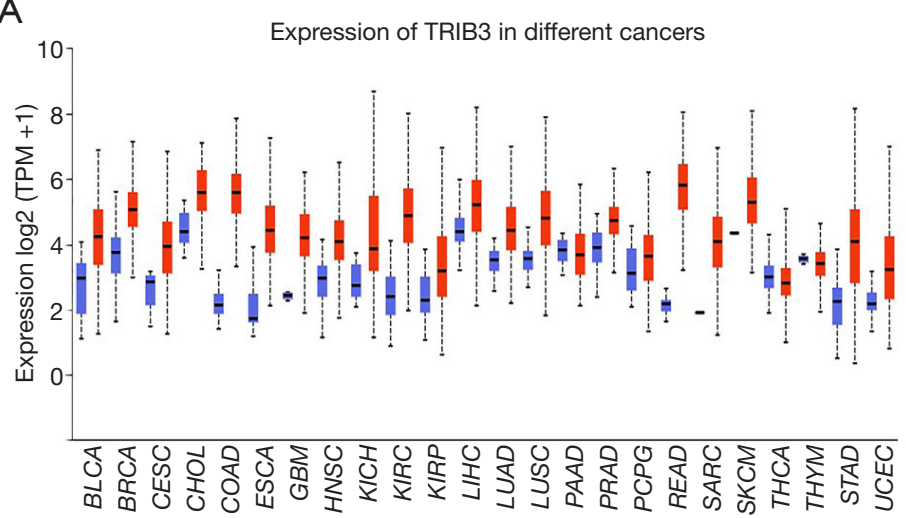

TCGA sample

C

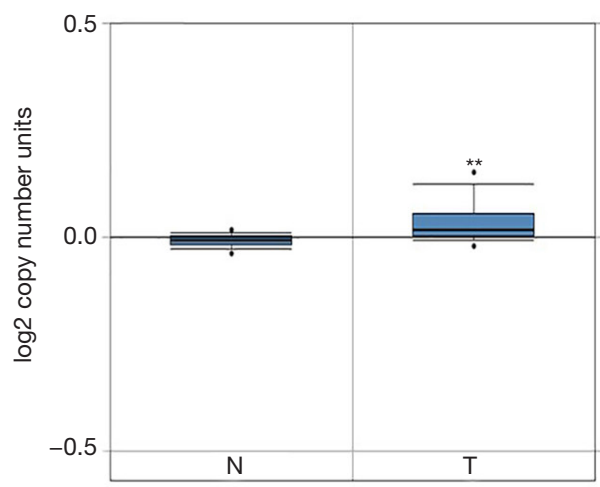

$\mathrm{E}$

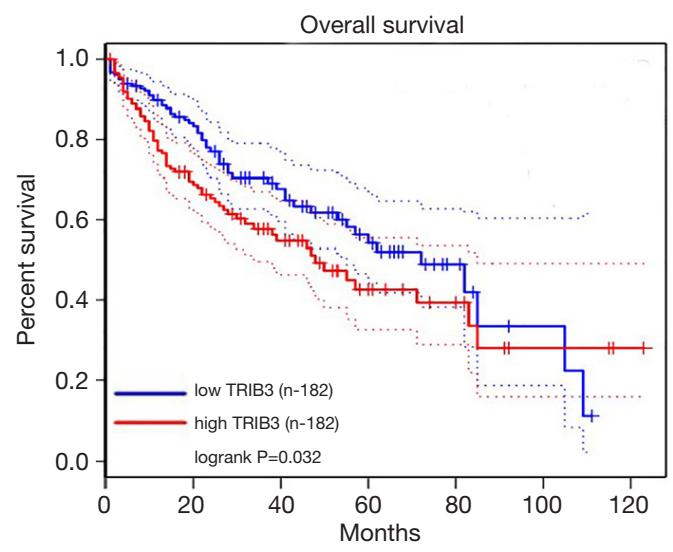

$\mathrm{F}$
B

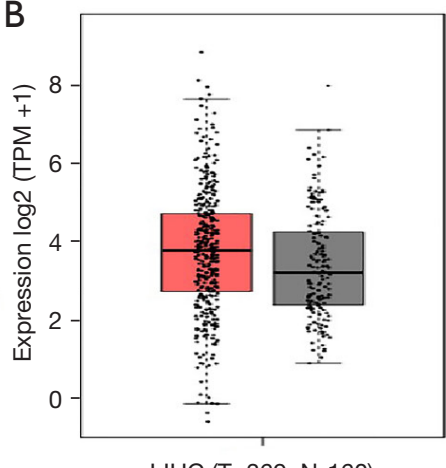

LIHC (T=369, N-160)
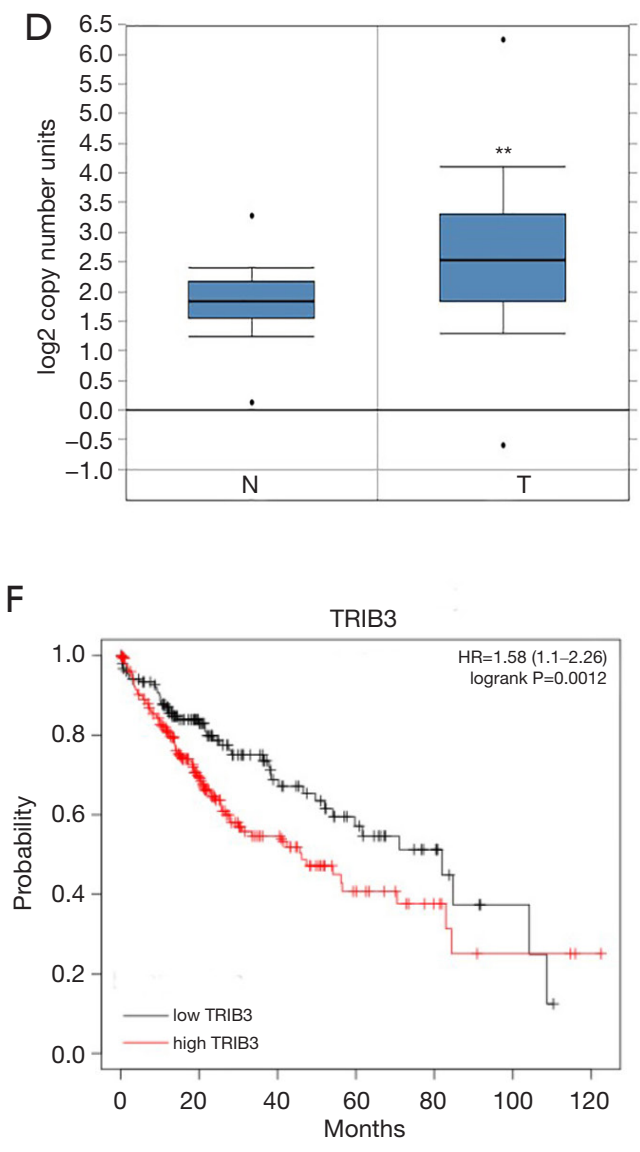

$\begin{array}{rllllll}\text { low } 154 & 85 & 42 & 24 & 11 & 3 & 0 \\ \text { high } 210 & 97 & 42 & 18 & 8 & 3 & 1\end{array}$

Figure 1 Increased expression of TRIB3 in LIHC tissues and this was associated with poor prognosis. (A) The expression of TRIB3 in different tumors; (B) in TCGA dataset, the expression of TRIB3 in LIHC tumor tissue was higher than that in normal tissues; (C,D) two datasets from Oncomine showed that TRIB3 is highly expressed in tumor tissues of HCCs; (E,F) K-M plot analysis showed that TRIB3 is related to the OS of HCC patients (data obtained from GEPIA and K-M plotter databases). **, $\mathrm{P}<0.01$. TRIB3, tribble pseudokinase 3; LIHC, liver hepatocellular carcinoma; TCGA, The Cancer Genome Atlas; HCCs, hepatocellular carcinomas; K-M, Kaplan-Meier; OS, overall survival. 
A

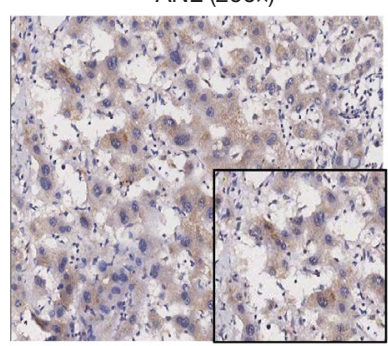

B
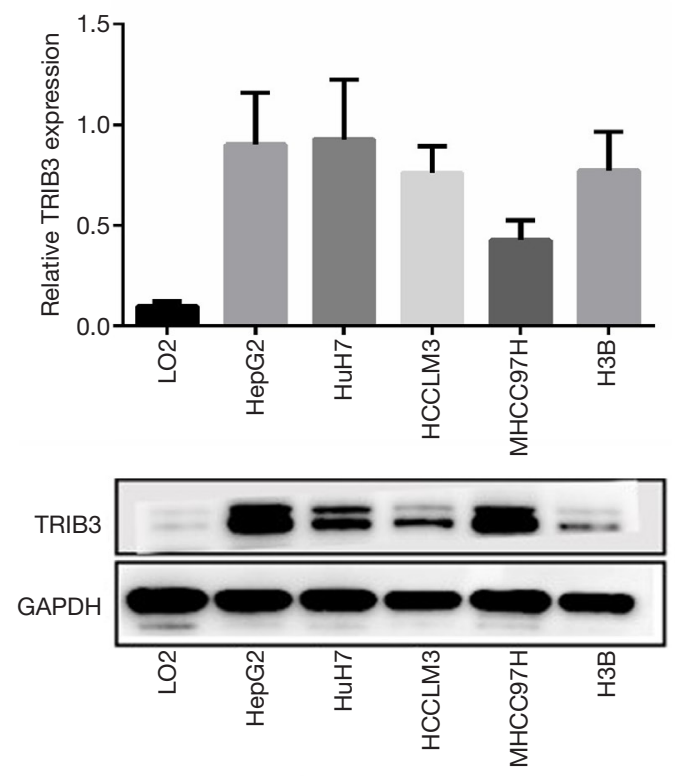

Stage I

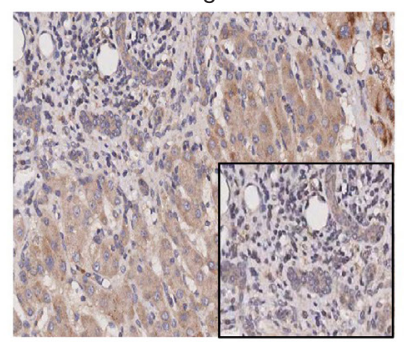

Stage II

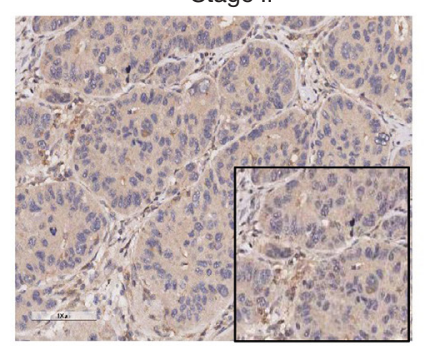

Stage III

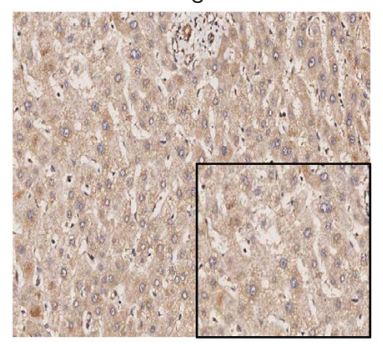

C

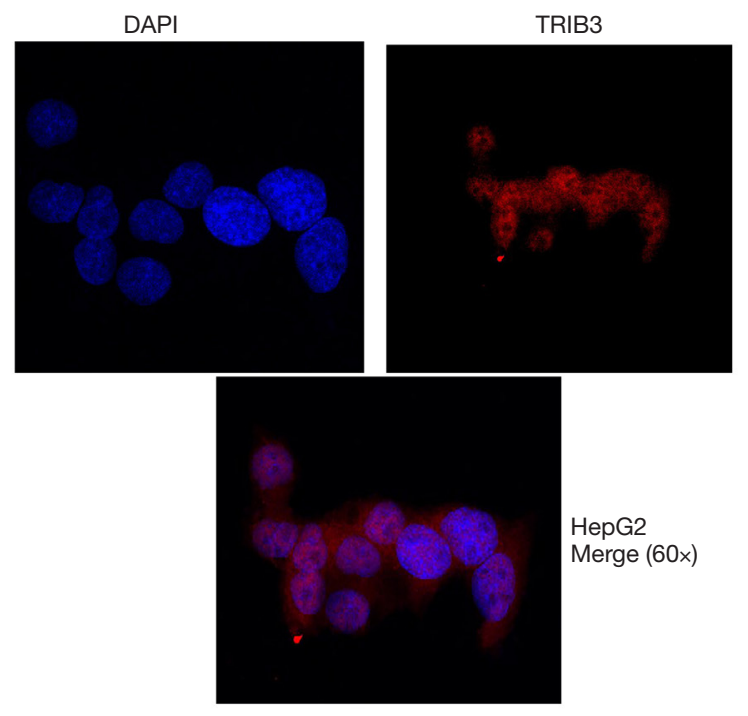

$E$

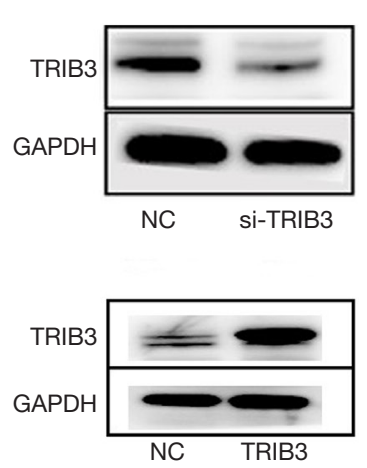

Figure 2 TRIB 3 is highly expressed in the advance stages of hepatocellular carcinoma and is mainly located in the cytoplasm. (A) Representative immunohistochemical images of TRIB3 expression in HCC at different stages of disease and in the adjacent normal liver tissues ( $\times 200$ ); (B) RT-qPCR and WBs were used to analyze the expression of TRIB3 in different HCC cell lines; (C) the subcellular localization of TRIB3 $(\times 60)$; (D,E) TRIB3 was knocked down (si-TRIB3) and overexpressed (TRIB3) in different cell lines and the effect was determined by RT-qPCR and WB. ${ }^{* * *}, \mathrm{P}<0.001$. TRIB3, tribble pseudokinase 3; ANL, adjacent normal liver; HCC, hepatocellular carcinoma; RT-qPCR, quantitative reverse transcription PCR; WB, Western blot; NC, negative control. 
A
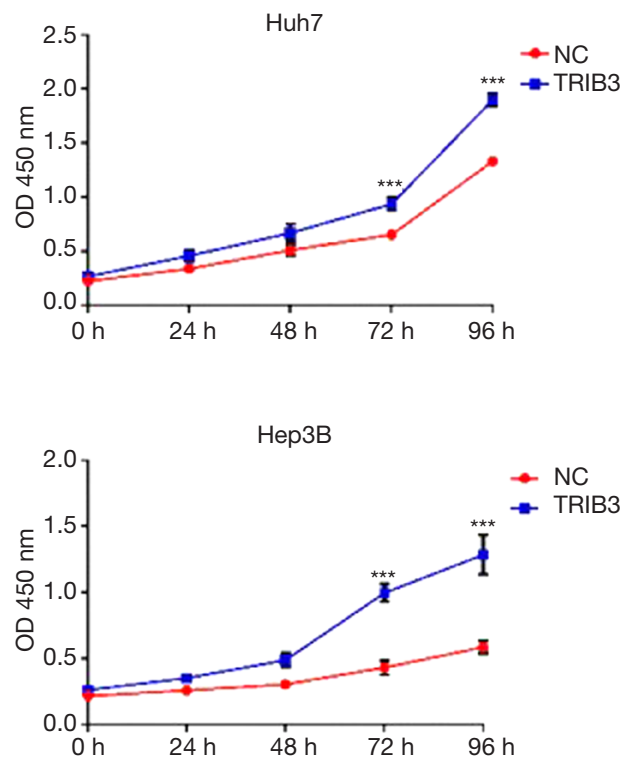
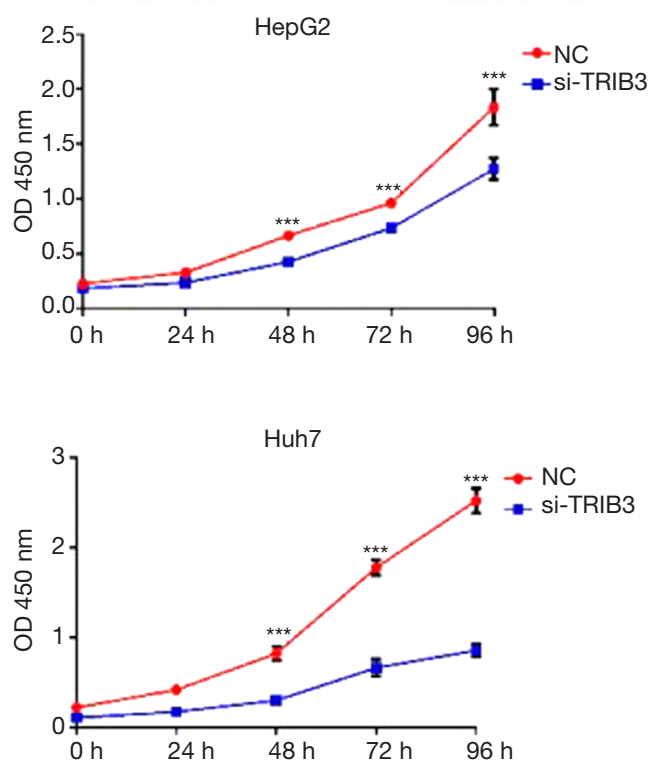

B

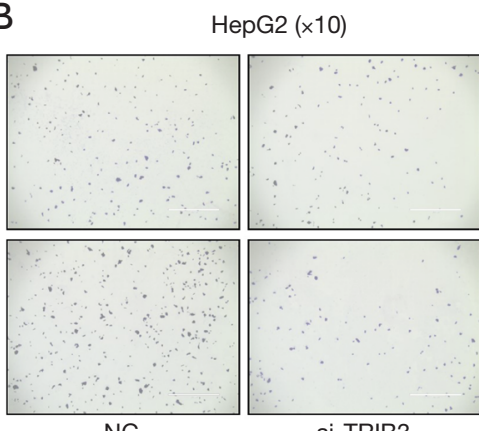

HepG2 ( $\times 10)$

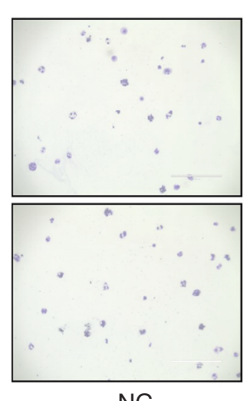

Huh7 $(\times 10)$
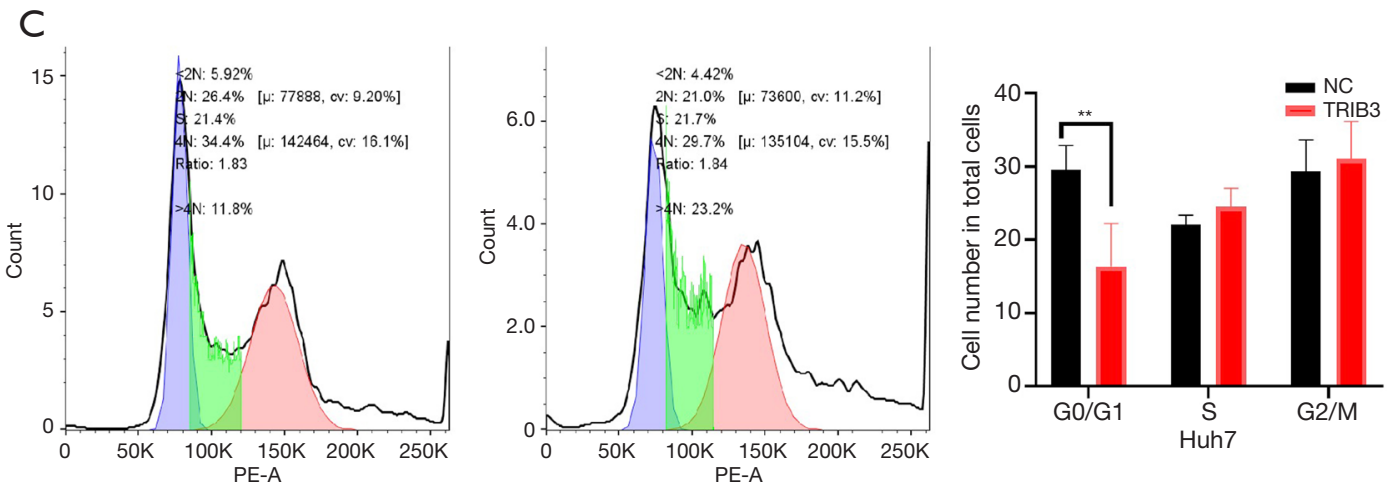

Figure 3 TRIB3 promotes the proliferation and cycle progression of hepatocellular carcinoma cells in vitro. (A) CCK8 analysis showed that overexpression of TRIB3 promoted the growth of Huh7 and Hep3B cells, while deletion of TRIB3 inhibited the growth of HepG2 and Huh7 cells; (B) clonogenic analysis Dyeing with crystal violet showed that the number of monoclones formed in HCC cells was related to the knockdown or overexpression of TRIB3 $(\times 10)$; (C) cell cycle analysis showed that in Huh7 cells, overexpression of TRIB3 and cycling decreased the G0/G1 fraction and increased the ratio of $\mathrm{S}$ and $\mathrm{G} 2 / \mathrm{m}$ in Hep3B cells. ${ }^{* *}, \mathrm{P}<0.01$; ${ }^{* * *}, \mathrm{P}<0.001$. TRIB3, tribble pseudokinase 3; HCC, hepatocellular carcinoma; NC, negative control. 
Huh7 cells identified a series of differentially expressed genes (DEGs) (Figure 4A). The gene set enrichment analysis (GSEA) showed that TRIB3 was associated with genes involved in the $M A P K$ signaling pathway (Figure $4 B$ ). The GEPIA 2.0 gene correlation analysis showed that the expression of TRIB3 was significantly positively correlated with the expression of $R A F\left(\mathrm{R}^{2}=0.22 ; \mathrm{P}<0.001\right), M E K$ $\left(\mathrm{R}^{2}=0.26 ; \mathrm{P}<0.001\right)$, and $E R K\left(\mathrm{R}^{2}=0.30, \mathrm{P}<0.001\right)$ (Figure $4 C$ ), all of which are involved in the MAPK signaling pathway that regulates a variety of cellular response mechanisms such as growth, proliferation, and metastasis. These three kinases can be activated to regulate cell growth, differentiation, stress adaptation to the environment, inflammation, and other vital cellular physiological and pathological processes. As shown by RT-qPCR and Western blotting, HepG2 cells transfected with si-TRIB3 showed significantly reduced levels of phosphorylated ERK (p-ERK) and phosphorylated MEK (p-MEK) (Figure 4D,4E). These data suggested that TRIB3 is an oncogene of HCC, which may affect ERK signaling pathways by interacting with these targets.

\section{Interaction between TRIB 3 and SMARCD 3}

Our previous study reported a close association between TRIB3 and SMARCD3 in the regulation of cardiovascular diseases (19). In patients with estrogen receptor (ER) positive breast cancer, high expression of SMARCD 3 is associated with improved survival (20). Interestingly, SMARCD 3 can promote tumor metastasis by activating cancer associated fibroblasts (CAFs) in colorectal cancer (21). The SWI/SNF (SWItch/Sucrose NonFermentable) chromatin remodeling factor SMARCD3/ baf60c has been shown to control epithelial mesenchymal transition by inducing Wnt5a signal transduction (22). However, to date, the interaction between SMARCD3 and TRIB 3 in LIHC has not been examined. Proteinprotein interaction (PPI) string prediction demonstrated a close correlation between TRIB 3 and SMARCD 3 (Figure $5 A$ ), and immunofluorescence staining revealed that they were co-localized in the nucleus of Huh7 cells (Figure 5B). Furthermore, co-IP assays using magnetic beads with the TRIB3 antibody showed that SMARCD3 bound specifically to TRIB3 (Figure 5C). Analyses using the GEPIA 2.0 database revealed a significant correlation between the two molecules $\left(\mathrm{R}^{2}=0.18 ; \mathrm{P}<0.001\right)$ (Figure $5 D$ ) and the overall survival of SMARCD3 was poor in HCC patients (Figure $5 E$ ), similar to that observed with TRIB3.

\section{Discussion}

While the incidence of HCC and the associated mortality rates have been somewhat alleviated with recent advances in diagnostic indicators and treatment methods, the prognosis for patients with HCC remains poor (23). This study demonstrated the carcinogenic role of TRIB 3 in HCC and its correlation with overall survival in patients with HCC. Silencing TRIB3 hindered the proliferation of hepatoma cells and inhibited the progression of the cell cycle. RNA-seq differential gene analyses demonstrated that overexpression of TRIB3 can activate the MAPK signaling pathway. Furthermore, TRIB3 and SMARCD3 were shown to interact with each other and co-localize in the nucleus of HCC cells. Our previous studies demonstrated that the interaction of TRIB3 and SMARCD3 may play a role in the regulation of cardiovascular generation (19). And indeed, this interaction may have a role in the tumorigenesis of liver cancer. Therefore, TRIB3 may be a prognostic biomarker for HCC and a potential target for HCC therapy.

There have been numerous reports in the literature examining the role of TRIB 3 in metabolic diseases such as cancer and cardiovascular disease (24). Abnormal expression of TRIB3 is related to the prognosis of colorectal cancer, prostate cancer, and breast cancer, however, its complex regulatory relationship and the molecular mechanisms in tumor cells have not been fully elucidated. In gastrointestinal tumors, TRIB 3 is highly expressed in gastric cancer cells, and is involved in VEGFR expression and tumor vascular density (14). Knockdown of TRIB3 downregulated VEGFR expression and endothelial cell recruitment, and inhibited tumor angiogenesis, suggesting that TRIB3 is associated with the regulation of tumor angiogenesis. In HCC, few studies have shown an association between TRIB 3 expression and tumor progression and prognosis (25). A variety of stress responses, such as nutritional deficiency, hypoxia, hyperglycemia and hyperinsulinemia, endoplasmic reticulum stress, and oxidative stress, can lead to upregulation of TRIB3 expression. In fact, TRIB3 is thought to be a key protein in the "stress response regulatory switch" (9).

TRIB3 can promote tumor progression by weakening the main intracellular degradation process called autophagy which has a preventive effect on early cancers (8). Studies have shown that the interaction between TRIB3 and p62/SQSTM1 can block autophagy, resulting in the 
A

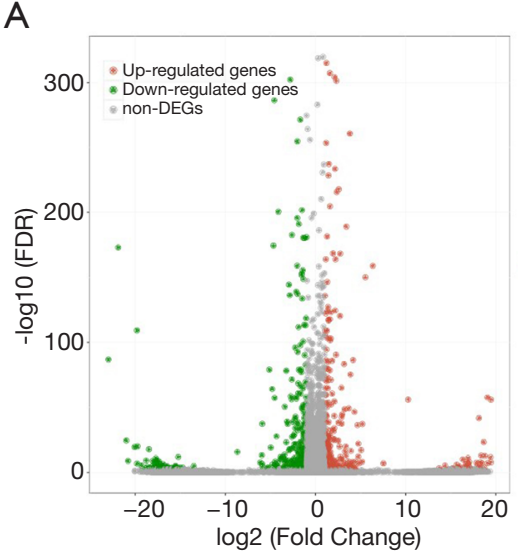

C

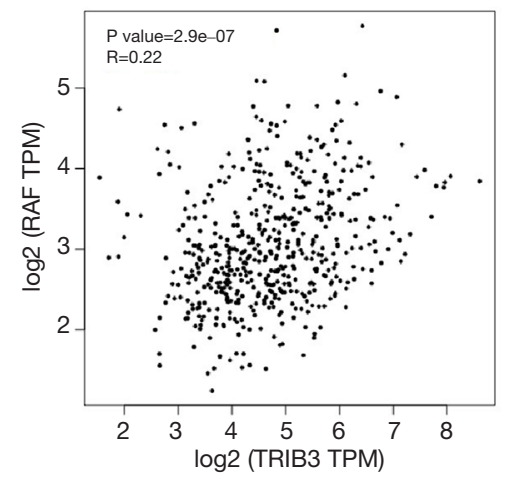

D
B
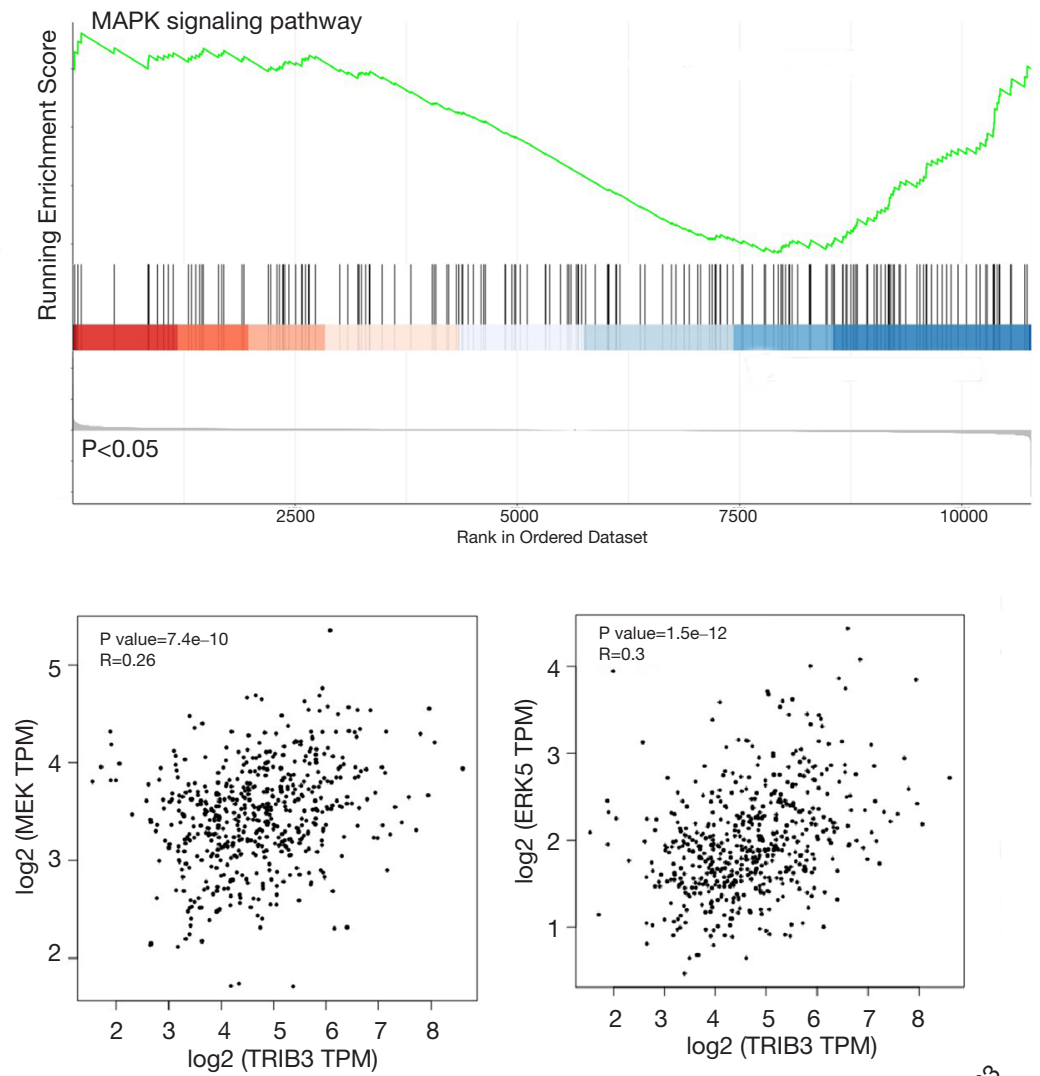

E
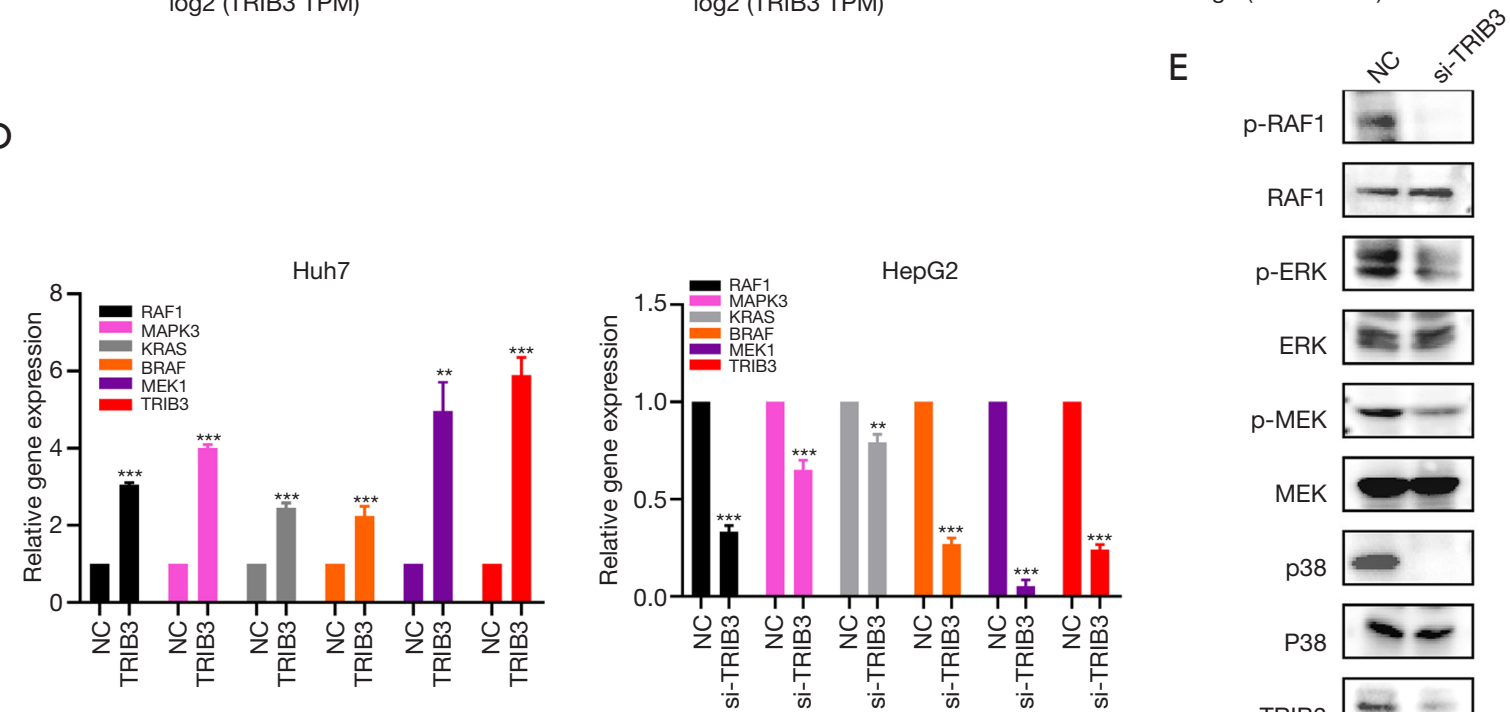

p-ERK $\geq 2$

ERK

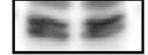

p-MEK

MEK

p38

P38

TRIB3

GAPDH
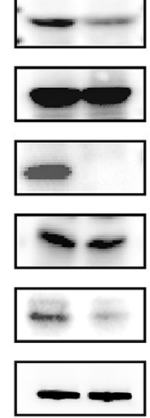

Figure 4 The role of TRIB3 in the MAPK pathway in tumor cells. (A) The volcano of DEGs in cells overexpressing TRIB3 compared to control cells; (B) the DEGs in cells overexpressing TRIB3 were enriched in the MAPK signaling pathway; (C) the RNA expression of RAF1, MAPK3, KRAS, BRAF, MEK1, and TRIB3 in cells overexpressing TRIB3 (TRIB3) compared to control cells (NC); (D,E) Western blot analysis showing the protein levels of p-RAF, RAF, p-ERK, ERK, p-MEK, MEK, p-38(MAPK), and P38, during knockdown of TRIB3. **, $\mathrm{P}<0.01$; ${ }^{* * *}, \mathrm{P}<0.001$. TRIB3, tribble pseudokinase 3; DEGs, differentially expressed genes; $\mathrm{p}-$, phosphorylated; NC, negative control. 
A

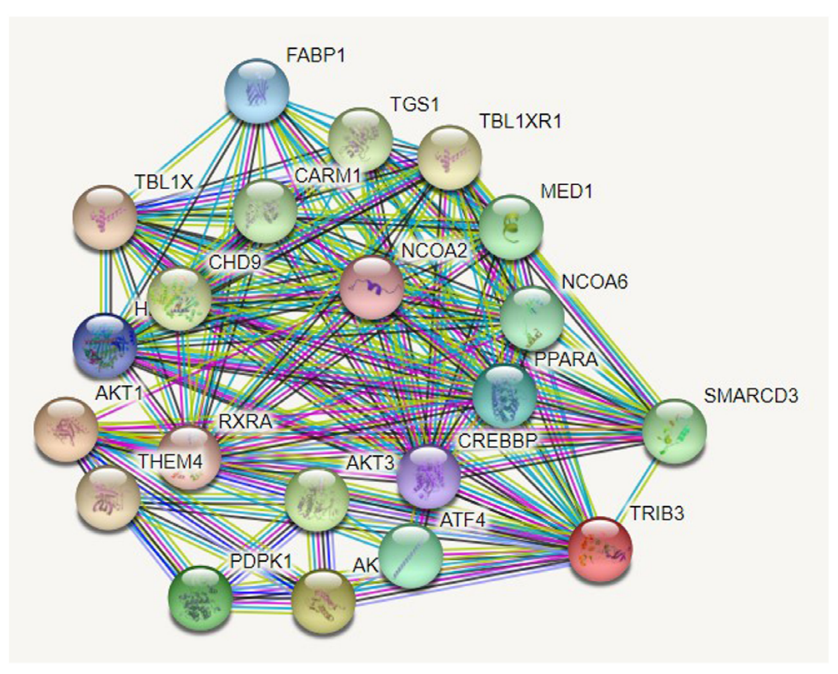

B

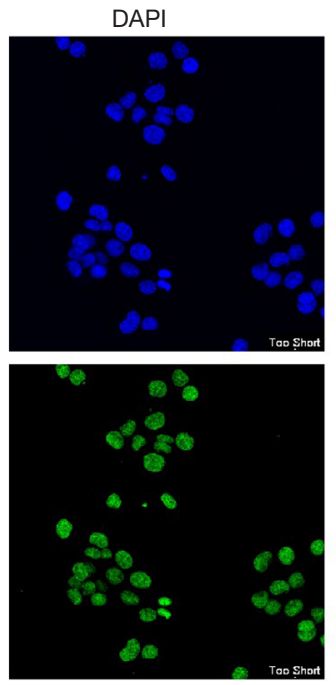

TRIB3
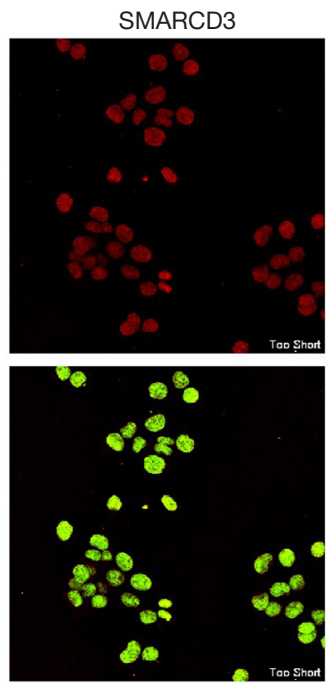

Merge $(\times 40)$
C

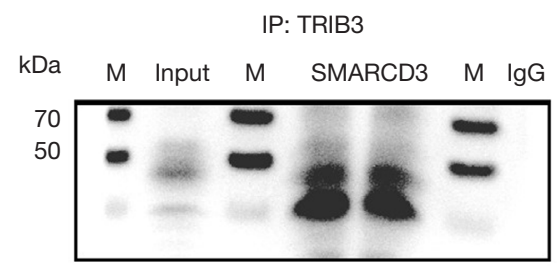

$\mathrm{D}$

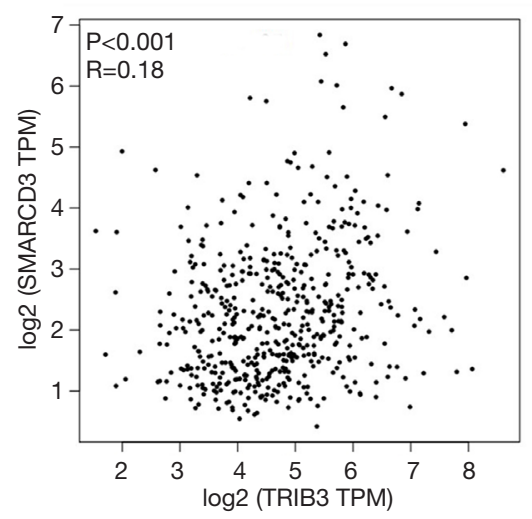

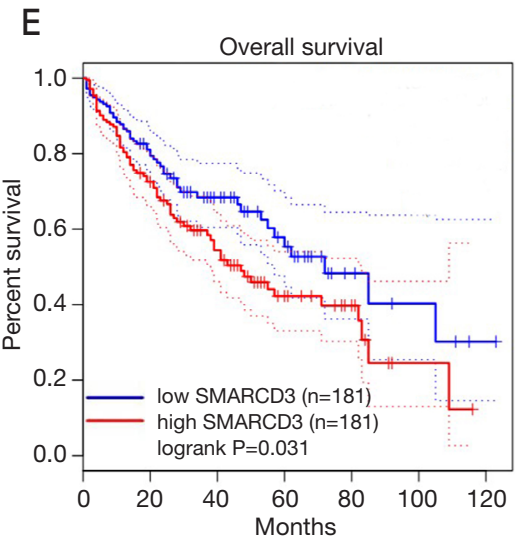

Figure 5 The correlation between TRIB3 and SMARCD3. (A) PPI String prediction showing an interaction between TRIB3 and SMARCD3; (B) immunofluorescence staining shows the co-localization of TRIB3 and SMARCD3 in the nucleus of Huh7 cells ( $\times 40)$; (C) Co-immunoprecipitation studies showing the interaction between TRIB3 and SMARCD3 in whole cell lysates; (D) The correlation between TRIB3 and SMARCD3 $\left(\mathrm{R}^{2}=0.18, \mathrm{P}<0.001\right)$; (E) Kaplan-Meier plot showing SMARCD3 expression in HCC and overall survival (data obtained from TCGA database). IP, immunoprecipitation; $M$, molecular marker.

accumulation of numerous tumor promoting factors. Blocking p62/TRIB3 interaction can attenuate the growth and metastasis of xenograft tumors, confirming the role of TRIB3 as an oncogene (26). In a recent study, high expression of TRIB3 was found in 176 samples which were associated with poor prognosis. Furthermore, the ability of TRIB3 to promote tumor development was confirmed in in vivo animal experiments (25). TRIB3 has also been shown to promote the progression of renal cell carcinoma cells via activating the MAPK signaling pathway (27).
This agrees with our current study showing that TRIB3 promoted tumorigenesis in liver cancer by activating the ERK signaling pathway.

MAPK signaling is a classic tumor research pathway, which can promote ERK phosphorylation into the nucleus and activate downstream gene transcription through signal activation. A recent study found that TRIB3 plays a role as a pseudokinase in the metabolic regulatory pathway of breast cancer cells. The pseudokinase and seeks domains can bind to a variety of transcription factors and signal 
proteins to inhibit the related regulatory pathways. TRIB3 and the MAPK-ERK/TGF were shown to play a leading role in regulating the notch regulatory pathway in breast cancer, and TRB 3 was suggested as a potential therapeutic target (28). Our current study confirmed that TRIB3 can promote the phosphorylation of this pathway, resulting in the proliferation of hepatoma cells. These studies all suggest a role for TRIB3 in different tumors and their related regulatory pathways.

The SMARCD 3 protein is a member of the SWI/SNF family which has ATPase activity (22). It can regulate the transcription of certain genes by changing the chromatin structure around the genes. Nonetheless, its biological function remains to be explored. Reports have suggested that SMARCD3 is a new prognostic biomarker and a potential therapeutic target in colorectal cancer, which may promote tumor metastasis by activating CAFs (21). Our previous study found a relationship between TRIM3, SMARCD3, and ATF6 gene mutations, and vascular complications in diabetic patients (19), however, the underlying mechanisms require further investigation.

This report demonstrated that TRIB 3 promotes the proliferation of HCC cells and enhances the progression of HCC. The precise molecular mechanisms by which TRIB3, $S M A R C D 3$, and other target molecules regulate liver cancer remain to be explored. Furthermore, the signal transduction pathways involved warrant further investigation. The results of this study support the application of TRIB3 as a diagnostic marker and as a potential therapeutic target in patients with HCC.

\section{Conclusions}

This investigation revealed that TRIB3 was highly expressed in the tissues of high-grade HCC, which is associated with poor prognosis in different cohorts. Furthermore, TRIB3 enhanced cell proliferation and accelerated the cell cycle, likely via its interaction with SMARCD3 and activation of the MAPK/ERK signaling pathway. TRIB3 and SMARCD3 were co-localized intracellularly and their expression was positively correlated. TRIB3 may represent a novel biomarker and therapeutic target for future diagnosis and treatment of liver cancer.

\section{Acknowledgments}

The authors would like to thank you Dr. Hui-Zi Li and Mei-Xiao Zhan for your constructive comments and suggestions prior to publication.

Funding: This study was funded by the Research Start Project of Zhuhai People's Hospital, China (No. 2020ycqd001).

\section{Footnote}

Reporting Checklist: The authors have completed the MDAR reporting checklist. Available at https://dx.doi. org/10.21037/atm-21-2820

Data Sharing Statement: Available at https://dx.doi. org/10.21037/atm-21-2820

Conflicts of Interest: All authors have completed the ICMJE uniform disclosure form (available at https://dx.doi. org/10.21037/atm-21-2820). The authors report funding support from the Research Start Project of Zhuhai People's Hospital, China (No. 2020ycqd001). The authors have no other conflicts of interest to declare.

Ethical Statement: The authors are accountable for all aspects of the work in ensuring that questions related to the accuracy or integrity of any part of the work are appropriately investigated and resolved. All procedures performed in this study involving human participants were in accordance with the Declaration of Helsinki (as revised in 2013). Written informed consent has been obtained from each patient and the study was approved by the ethics committee of Zhuhai People's Hospital, Zhuhai Hospital Affiliated with Jinan University.

Open Access Statement: This is an Open Access article distributed in accordance with the Creative Commons Attribution-NonCommercial-NoDerivs 4.0 International License (CC BY-NC-ND 4.0), which permits the noncommercial replication and distribution of the article with the strict proviso that no changes or edits are made and the original work is properly cited (including links to both the formal publication through the relevant DOI and the license). See: https://creativecommons.org/licenses/by-nc$\mathrm{nd} / 4.0 /$.

\section{References}

1. Maluccio M, Covey A. Recent progress in understanding, diagnosing, and treating hepatocellular carcinoma. CA Cancer J Clin 2012;62:394-9. 
2. Sung H, Ferlay J, Siegel RL, et al. Global Cancer Statistics 2020: GLOBOCAN Estimates of Incidence and Mortality Worldwide for 36 Cancers in 185 Countries. CA Cancer J Clin 2021;71:209-49.

3. Llovet JM, Kelley RK, Villanueva A, et al. Hepatocellular carcinoma. Nat Rev Dis Primers 2021;7:6.

4. Debes JD, Romagnoli PA, Prieto J, et al. Serum

Biomarkers for the Prediction of Hepatocellular Carcinoma. Cancers (Basel) 2021; 13:1681.

5. Eyers PA, Keeshan K, Kannan N. Tribbles in the 21st Century: The Evolving Roles of Tribbles Pseudokinases in Biology and Disease. Trends Cell Biol 2017;27:284-98.

6. Muscari F, Maulat C. Preoperative alpha-fetoprotein (AFP) in hepatocellular carcinoma (HCC): is this 50-year biomarker still up-to-date? Transl Gastroenterol Hepatol 2020;5:46.

7. Salazar M, Lorente M, Orea-Soufi A, et al. Oncosuppressive functions of tribbles pseudokinase 3 . Biochem Soc Trans 2015;43:1122-6.

8. He F, Sun B, Li L, et al. TRIB3 rs6037475 is a potential biomarker for predicting felodipine drug response in Chinese patients with hypertension. Ann Transl Med 2020;8:437.

9. Richmond L, Keeshan K. Pseudokinases: a tribble-edged sword. FEBS J 2020;287:4170-82.

10. Hua F, Shang S, Yang YW, et al. TRIB3 Interacts With $\beta$-Catenin and TCF4 to Increase Stem Cell Features of Colorectal Cancer Stem Cells and Tumorigenesis. Gastroenterology 2019;156:708-721.e15.

11. Wennemers M, Bussink J, Grebenchtchikov N, et al. TRIB3 protein denotes a good prognosis in breast cancer patients and is associated with hypoxia sensitivity. Radiother Oncol 2011;101:198-202.

12. Yu JM, Sun W, Wang ZH, et al. TRIB3 supports breast cancer stemness by suppressing FOXO1 degradation and enhancing SOX2 transcription. Nat Commun 2019;10:5720.

13. Ma B, Zhang H, Wang Y, et al. Corosolic acid, a natural triterpenoid, induces ER stress-dependent apoptosis in human castration resistant prostate cancer cells via activation of IRE-1/JNK, PERK/CHOP and TRIB3. J Exp Clin Cancer Res 2018;37:210.

14. Dong S, Xia J, Wang H, et al. Overexpression of TRIB3 promotes angiogenesis in human gastric cancer. Oncol Rep 2016;36:2339-48.
15. Yu JJ, Zhou DD, Yang XX, et al. TRIB3-EGFR interaction promotes lung cancer progression and defines a therapeutic target. Nat Commun 2020;11:3660.

16. Felip I, Moiola CP, Megino-Luque C, et al. Therapeutic potential of the new TRIB3-mediated cell autophagy anticancer drug ABTL0812 in endometrial cancer. Gynecol Oncol 2019;153:425-35.

17. Wang S, Wang C, Li X, et al. Down-regulation of TRIB3 inhibits the progression of ovarian cancer via MEK/ERK signaling pathway. Cancer Cell Int 2020;20:418.

18. Wang RQ, Long XR, Zhou NN, et al. Lnc-GAN1 expression is associated with good survival and suppresses tumor progression by sponging mir-26a-5p to activate PTEN signaling in non-small cell lung cancer. J Exp Clin Cancer Res 2021;40:9.

19. He F, Shu Y, Wang X, et al. Intensive Glucose Control Reduces the Risk Effect of TRIB3, SMARCD3, and ATF6 Genetic Variation on Diabetic Vascular Complications. Front Pharmacol 2018;9:1422.

20. Tropée R, de la Peña Avalos B, Gough M, et al. The SWI/ SNF subunit SMARCD3 regulates cell cycle progression and predicts survival outcome in ER+ breast cancer. Breast Cancer Res Treat 2021;185:601-14.

21. Jiang M, Wang H, Chen H, et al. SMARCD3 is a potential prognostic marker and therapeutic target in CAFs. Aging (Albany NY) 2020;12:20835-61.

22. Jordan NV, Prat A, Abell AN, et al. SWI/SNF chromatinremodeling factor Smarcd3/Baf60c controls epithelialmesenchymal transition by inducing Wnt5a signaling. Mol Cell Biol 2013;33:3011-25.

23. Lu J, Zhong BY, Zhu HD, et al. Embolotherapy of unresectable hepatocellular carcinoma: Eastern perspective. Chin Clin Oncol 2019;8:60.

24. Prudente S, Sesti G, Pandolfi A, et al. The mammalian tribbles homolog TRIB3, glucose homeostasis, and cardiovascular diseases. Endocr Rev 2012;33:526-46.

25. Wang XJ, Li FF, Zhang YJ, et al. TRIB3 promotes hepatocellular carcinoma growth and predicts poor prognosis. Cancer Biomark 2020;29:307-15.

26. Hua F, Li K, Yu JJ, et al. The TRIB3-SQSTM1 interaction mediates metabolic stress-promoted tumorigenesis and progression via suppressing autophagic and proteasomal degradation. Autophagy 2015;11:1929-31. 
27. Hong B, Zhou J, Ma K, et al. TRIB3 Promotes the Proliferation and Invasion of Renal Cell Carcinoma Cells via Activating MAPK Signaling Pathway. Int J Biol Sci 2019;15:587-97.

Cite this article as: Wang RQ, He FZ, Meng Q, Lin WJ, Dong JM, Yang HK, Yang Y, Zhao M, Qiu WT, Xin YJ, Zhou ZL. Tribbles pseudokinase 3 (TRIB3) contributes to the progression of hepatocellular carcinoma by activating the mitogen-activated protein kinase pathway. Ann Transl Med 2021;9(15):1253. doi: 10.21037/atm-21-2820
28. Izrailit J, Berman HK, Datti A, et al. High throughput kinase inhibitor screens reveal TRB3 and MAPK-ERK/ TGF $\beta$ pathways as fundamental Notch regulators in breast cancer. Proc Natl Acad Sci U S A 2013;110:1714-9. 
Supplementary

Table S1 The siRNA and primer sequences of TRIB3

\begin{tabular}{lcc}
\hline siRNA or primer & Sense (5'-3') & Antisense (5'-3') \\
\hline Human TRIB3 siRNA & CGGTTGGAGTTGGATGACAACTTAG & GATCTCAAGCTGTGTCGCTTT \\
Human TRIB3 & GCCACTGCCTCCCGTCTTG & GCTGCCTTGCCCGAGTATGA \\
Human GAPDH & CATCATCCCTGCCTCTACTGG & CTGGTGAATTTACCGGGAAG \\
\hline
\end{tabular}
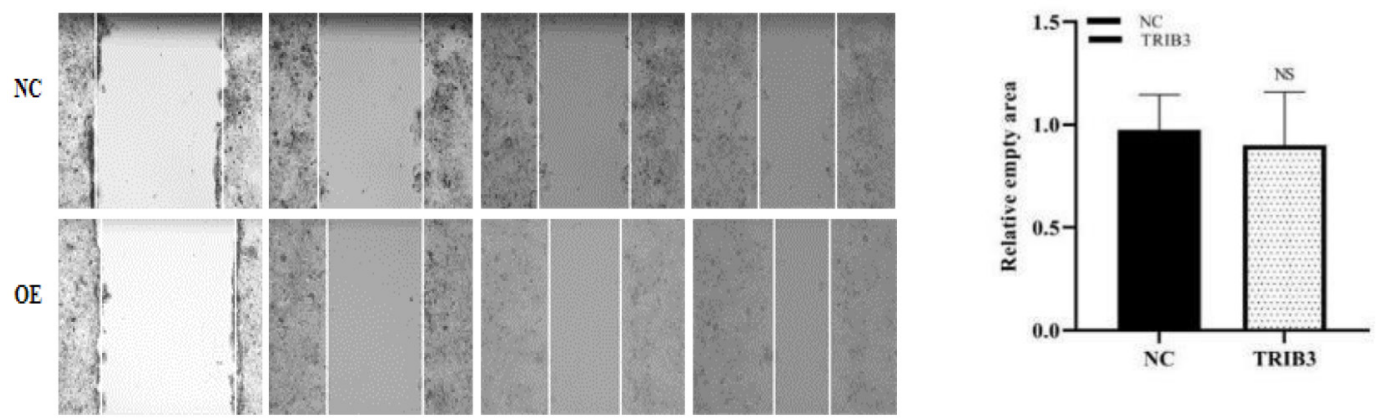

Figure S1 TRIB3 did not affect cell migration detected by wound healing assays in Hep3B cells. 